\title{
Roundabout perception: review of standards and guidelines for advanced warning
}

\author{
A. Pratelli ${ }^{1}$, R. R. Souleyrette ${ }^{2}$ \& C. Harding ${ }^{3}$ \\ 'Dipartimento di Ingegneria Civile "Vie e Trasporti", \\ University of Pisa, Italy \\ ${ }^{2}$ Department of Civil, Construction and Environmental Engineering, \\ Iowa State University, USA \\ ${ }^{3}$ Human Computer Interaction Program, Iowa State University, USA
}

\begin{abstract}
This paper deals with roundabout visibility and perception design requirements, including available standards and guidelines in the US and Europe for warning signs. To provide for the highest levels of safety, visual perception of junctions is important even where advance warning signs are provided, to reinforce driver knowledge and confidence in their actions. For roundabouts, three different types of central island development are recently suggested to affect their visual perception, and the related classification defines the reduced, the compressed and the streamlined, or slender, central island development. The paper proposes new methodologies to test this perceptibility of various roundabout designs and developments. Two research strategies based on the specific roundabout visual intrusion defined by the solid angle are outlined: on-site stand-by/2D and virtual video/3D. Finally, some preliminary results are discussed in view of selecting original and proper standards. Moreover, future research is suggested for extending the methodology to more comprehensive application.
\end{abstract}

Keywords: roundabout design and safety, roundabout perceptibility, driver visual perception, solid angle, visual intrusion, central island development.

\section{Introduction}

The design of a roundabout, like that of any other road component, should be mainly based on principles of safety and capacity. But, in addition to using geometric standards, performance formulas and capacity models, many aspects 
of perception and visual appeal should be also considered in a systemic design context. Therefore, it is important to consider human factors and driving behaviour. As with all types of intersections, including roundabouts, practical experience indicates four basic safety and operational considerations, namely: clarity, visibility, comprehensibility and space for design vehicles.

The perceptibility of road features is known to be highly related to safety performances [1,2]. Warning signs may be provided to call attention to road features where driver perception may be limited or more important. This is often the case for curves, or decision points such as intersections, or merges points. Warning and perception may be especially important for certain road elements such as roundabouts where users may be unfamiliar with modern (priority-tocircle) roundabouts.

Following Pratelli and Souleyrette [3], the three basic types of central island development can be recognized and classified as follows:

a) Reduced: which means a development spread and diffused over the whole surface of the inner island. This type includes simple green "hill", or lawn adorned with seasonal flowers and low bushes. The practical threshold for the reduced treatment height is $1.25 \mathrm{~m}$ above road pavement;

b) Compressed: are set to "compressed volume" those treatments made with thick clumps of a certain height, more than 1,25 meters or low trees with voluminous hair, or sculptures of horizontal and vertical dimensions not much different. It can be defined as "compressed" or "squat" a shape that is inscribed in a rectangular base ratio greater height not exceeding 1,5.

c) Slender: central island treatments of the slender type are designed with a prevalent central element, for example, a tree canopy, a tall thin sculpture or an attractive pole lighting. The slender type is referred to a virtual circumscribed box to the inner apparels which have its major base length 5 to 7 times less than the central island diameter and its height is great or equal to 3 to 4 times of the previous major base.

The following sections outline how different central island developments could be appraised for a driver looking at a roundabout from an approaching lane, and how solid angle based methods could be applied to visual intrusion in a safety oriented design way.

\section{The driver visual perception behaviour}

All drivers make their driving actions and decisions as a sequence of manoeuvres which are responses to different stimuli received and perceived both from road geometry and outside environment. Then it is obvious that a driver must be able to see in order to drive.

Nevertheless, relationships between visual acuity and driving behaviour are not readily specified. It is well known that on a modern roundabout, deflection trajectories force drivers to reduce their speed, leading to both lower probability of severity of crashes [4]. 


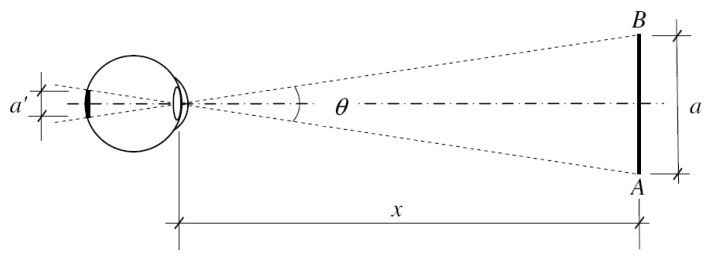

Figure 1: Image formed on retina of the driver when he looks at a given object.

As a result of deflection on a trajectory curve the vehicle is subject to heel because of centrifugal force and the driver is consequently led to combat it by reducing the speed and at the same time you obtain a greater attention to driving.

Another important human stimulus arising in vehicle driving is visual perception of the relative speed referred to fixed or moving obstacles placed on the road or on its lateral sides.

When a driver is on a vehicle at high speed, his attention is turned to the motion trajectory and the eye rotation is very limited. In fact, the visual field is lowered as much as the speed grows. For instance, the clear vision field angle is about 3 degree for speeds ranging from 80 to $100 \mathrm{~km} / \mathrm{h}$, and the out of focus vision field is about 45 degree. Dimension $a^{\prime}$ is the image formed on retina of the driver when he looks at a given object $\mathrm{AB}$, and it is proportional to the value $\theta$ of the angle by which the object is viewed and having its vertex in the centre of the eye (Figure 1). Any change in distance $x$ implies a variation in value of $\theta$, and therefore the image dimension changes with angular speed $\dot{\theta}$ :

$$
\dot{\theta}=\mathrm{d}(\mathrm{a} / \mathrm{x}) / \mathrm{dt}=a \cdot v / x^{2}
$$

If such an angular speed $\dot{\theta}$ crosses above a critical threshold, then the driver have a perception of danger, due to the fact he has the illusion of colliding with the object. By consequence, the driver reacts in such a manner to maintain $\dot{\theta}$ at endurable values below critical threshold. This last varies from driver to driver and its average value has been experimentally measured in 3 to $5 \cdot 10^{-3} \mathrm{rad} / \mathrm{sec}$.

Now, let us consider a vehicle moving at speed $v$ on rectilinear road track where there is an obstacle, e.g. a road sign, placed on the road side at distance $d$ from the driver's eye trajectory.

Figure 2 depicts both angle $2 \varphi$, which is the clear visual field angle relate to speed $v$, and the distance $x$ between the driver's eye and the obstacle. The obstacle goes out of the clear visual field, i.e. the driver has an out of focus vision, at any value of $d$ below:

$$
d=x \cdot \tan \varphi
$$

Equation (2) can be resembled to the straight line in Figure 3. On the other side, the distance $x$ below dimension $a$ ' of the object image on driver's eye retina 


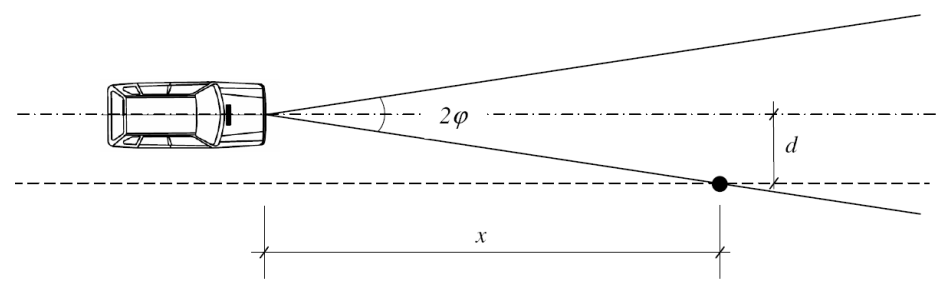

Figure 2: Distance $x$ between the driver's eye and an obstacle on the road side at distance $d$ from the driver's eye trajectory.

changing at angular speed values that he is not able to tolerate, is obtained from (1) assigning to $\dot{\theta}$ the critical threshold value. Continuous and dotted line curves in Figure 3 depict this relation existing between the two dimensions $d$ and $x$. The diagram of Figure 3 shows that any object placed on the road lateral side falls inside or outside the clear vision field if the two points related to its own longitudinal distance $x$ and transversal distance $d$ are located above or below the straight line with slope $\varphi$. If the vehicle has speed $v$, the angular speed at which the image on driver's eye retina changes its dimensions, may be, or be not, an annoyance for the driver depending to the point of $x$ and $d$ is above, or below, the curve related to $v$.

Therefore, the dangerous zone in the diagram of Figure 3 is the dotted one, comprised between the straight line and the curve related to speed $v$. Every point falling into such a danger zone is referred to a situation of an object falling into the clear vision field and at the same time the image dimension $a$ ' of its lateral distance $d$ varies with an angular speed $\dot{\theta}$ above the critical threshold.

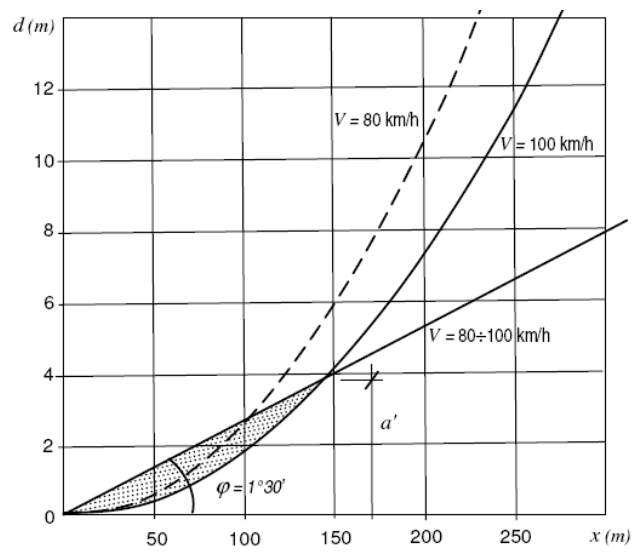

Figure 3: Distance $x$ between the driver's eye and an obstacle on the road side at distance $d$ from the driver's eye trajectory.

When an obstacle is placed at a distance below ordinate $a^{\prime}$ of the intersection point between the straight line with slope $\varphi$ and the curve related to vehicle 
speed $v$, as the vehicle is approaching to the obstacle it is reached a distance $x$ corresponding to the point entrance into the danger zone. In such a situation, the driver reacts to the unpleasant sensation of danger increasing his distance $d$ from the obstacle. The driver can steer left or slow down its vehicle speed, and each one of these two manoeuvres leads to move the representative point out of the danger zone.

Similarly, when a faster vehicle reaches another preceding vehicle on the same lane. Distance $x$ between the two vehicles becomes to reduce progressively, while angular speed $\dot{\theta}$ progressively increases. When $\dot{\theta}$ crosses above the threshold value, the driver of the oncoming vehicle perceives the sensation of danger and he reacts slowing down his vehicle speed or, if possible, changing lane to overpass.

\section{Perception of signs and roundabouts}

In today's increasingly complex driving environment, signs need to be easily detected and understood at a glance. They warn, inform and regulate drivers. Road authorities in North America and Europe have often improved signs by using larger and more legible fonts and better materials to increase their visibility. Nevertheless, a key to providing a safe and efficient driving environment is to take a proactive approach and provide the driver with necessary information in the safest and most effective way possible, while minimizing distraction.

Drivers often need to quickly process a great deal of information. The driver must be able to glance at the sign and rapidly determine whether the information is relevant and if so, needs to be processed, retained and acted upon. Signs, for example, have to be read within the 3 to 5 degree clear vision field. Their placement and design should allow this to occur without forcing drivers to divert their vision from the roadway ahead [5].

An important aspect of roundabout design is the provision of warning signs placed at an adequate sight distance for all approaches, as above. Warning signs call attention to unexpected conditions on adjacent to a highway or street and to situations that might call for a reduction of speed or an action in the interest of safety and efficient traffic operations. The Manual on Uniform Traffic Control Devices [6] specifies some requirements and distance positioning tables for design and practice.

Moreover, another important aspect of visibility is the ability of drivers to perceive or recognize the main features of the roundabout, i.e. the central island and the splitter and raised islands. The "legibility" of a roundabout can be defined by a set of unmistakable and peculiar factors that are visible and contribute to the driver's recognition of the intersection. Pertinent and specific regulations on roundabout legibility and recognition can be found in recent provisions such as Swiss SN guidelines [7] and French CERTU standards [2].

In modern roundabouts, where traffic yields on entry, the central island may be furnished with elements of significant size such as trees, large bushes, or 
artistic features which are generally considered obstructions to sight distance for safe movement.

A driver entering the roundabout in fact should be concentrating his or her attention to the left and not straight through the roundabout. A driver within the roundabout should then turn his or her attention to what is the front and to the right. In neither of these two cases should the driver focus attention on the other side of the roundabout.

The details of the roundabout and a distinctive central island are therefore very important for safety by warning the drivers through a good perception and recognition at a distance from the intersection. Therefore, the central island, if developed in a particularly distinctive manner, may enhance both the safety of traffic operations and the effects of moderation of speeds [3].

\section{The safety side of visual intrusion}

Visual intrusion has been initially conceived as a negative factor of transport facilities and it was seen as a change of view generally affecting the quality of the visual environment [8]. Nevertheless, such a definition not necessarily implies a negative way of judgement. There are cases where the insertion of a new element has a visual decorative effect and therefore it is positive.

Visual intrusion is strictly related to properties of the human eye, which have been sketched above, point to the fact that objects which are seen centrally convey more "information" in term of detail than objects which are seen peripherally. The clear visual field is therefore more important in assessing the intrusive effect of, for example, an elevated urban highway.

Visual intrusion can be quantified as a function of the solid angular subtended of the intruding artefact, modified by a position factor based upon the position of the artefact in the visual field [8]. The solid angle, $\Omega$, is the angle in threedimensional space that an object subtends at a point. It is a measure of how big that object appears to an observer looking from that point. The observer position is a crucial factor of the measure: a small object nearby could subtend the same solid angle as a large object far away.

The solid angle is proportional to the surface area, $S$, of a projection of that object onto a sphere centred at that point, divided by the square of the sphere's radius, $R$ : i.e., $\Omega=S / R^{2}$. A solid angle is related to the surface of a sphere in the same way an ordinary angle is related to the circumference of a circle [9]. It is measured in steradians, or "sr", and the solid angle corresponding to all of the space being subtended is $4 \pi$ steradians.

Now, one can think of using visual intrusion in a positive way, as a design tool for enhancing driver awareness and perception of a roundabout. In order to evaluate the visual obstruction of a given type of the central island development, one may refers its related solid angle to a standard distance also usually found in road construction guidelines and traffic safety handbooks, e.g. the distance before the yield line from which the minimum clear sight distance must be provided or the minimum safe-stopping distance before the yield line. 


\section{The on-site stand-by/2D approach}

We call "on-site stand-by/2D approach" the following method based on instrumental field observations which are currently in progress.

Solid angle and geometrical field data are under collection on a large sample of different roundabouts using a laser rangefinder Leica A8, which is placed in the middle of every entrance lane at the distance of 15 meters from the markings of yield line and at the height of 1.25 meters from road pavement. These values of the instrumental position are selected due to the fact that one is the minimum clear sight distance and the other is often referred as the average standard height of driver's eye.

The laser rangefinder Leica A8 is a longimetric indirect instrument to accurately measure lengths, areas and volumes. It is characterized by the fact that emits a laser beam directed at the measuring point and can be used up to distances of 100 meters. From the instrumental point we are collecting measures, or deriving them through mathematical formulas, related to the distinctive geometrical characteristics and visual intrusion appraisal of each roundabout (inscribed circle diameter, central island diameter, carriageway width, surface portion of treatment framed to calculate the solid angle).

The ultimate goal is to find the experimental calibration of the roundabout classification model quoted above which is based on the three suggested standard development types of the roundabout central island [3]. We trust to present our extensive research findings and results in a forthcoming paper.

\section{The virtual video/3D approach}

Our empirical "video/3D", or proof-of-concept, research approach investigates the relationship between a roundabout's treatment type and size - central island development profile [3] - and its perceptibility.

Looking at 40 roundabouts located in Italy, we have linked the (geometric) concept of the solid angle to the type of treatment as classified into reduced, compressed or slender. Using a similar approach, we investigate 43 roundabouts in the US based on a "virtual" methodology.

We use Google Earth and Google Streetview to measure the base, height and diameter of each treatment, calculate their areas, base-to-height ratios and solid angles at different distances, as well as classify by treatment type. In addition to these geometric concepts we also involve a form of perceptibly as a way of establishing how well a driver perceives different types and profiles of treatments. Perceptional psychology establishes a complex framework for statements about human perception [10]. Our work simply defines perceptibility as the distance at which a driver is reasonably sure that a generic obstacle/obstruction and closer, more specifically, a roundabout is ahead.

Our aim is to correlate this human-derived measurement with treatment type and profile in order to derive some indication on its effectiveness in communication the presence of a roundabout. To investigate this concept we conducted an informal user study which "simulates" the driver approaching a 

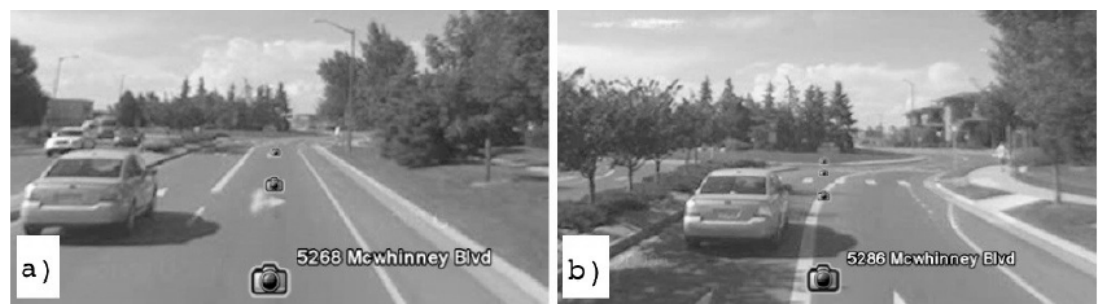

Figure 4: Two "Streetview" views of roundabout approach, a) Ro, the "first indication" of an obstacle and b) $R r$, the certain recognition of a roundabout with forest as treatment.

specific roundabout via Google Streetview, a 360-degree snapshot taken roughly every 10 to 15 meters. In our proof-of-concept research we performed an informal user study to measure perceptibility as two different distances:

a) $R o$ for an early indication of an upcoming (approaching) obstacle;

b) $R r$ for the clear awareness that a roundabout is ahead.

Figure 4 illustrates both distances on an approach to a large roundabout with a center island (treatment is a grove of trees). In Figure 4.a) the distance of the driver (Streetview camera) to the treatment is $108 \mathrm{~m}$; for Figure 4.b) the distance is 56 meters, the camera icons show the closest Streetview locations.

These distances are the result of a purely visual judgment of recognition; street signs officially announcing the roundabout were deliberately ignored. For a driver unfamiliar with the area, $R o$ would represent a distance where it is clear that something unusual (a potential obstacle) is ahead, but only at $R r$ would the driver be reasonably certain that a roundabout is ahead and react accordingly.

\subsection{Proof-of-concept method and results}

Starting with a list of US roundabouts obtained from the website http://roundabout.kittelson.com/Roundabouts, we developed a subset of 43 roundabouts approaches suitable for our purposes.

Using Google Maps, we selected roundabouts with one or more straight (tangent), at least $300 \mathrm{~m}$ long approaches, where Google Streetview was available and where relatively few obstructions (other vehicles, overhanging trees) were present (Figure 5). For each roundabout, we measure the outside diameter, $D$, including the circulating road and the width of the base of the

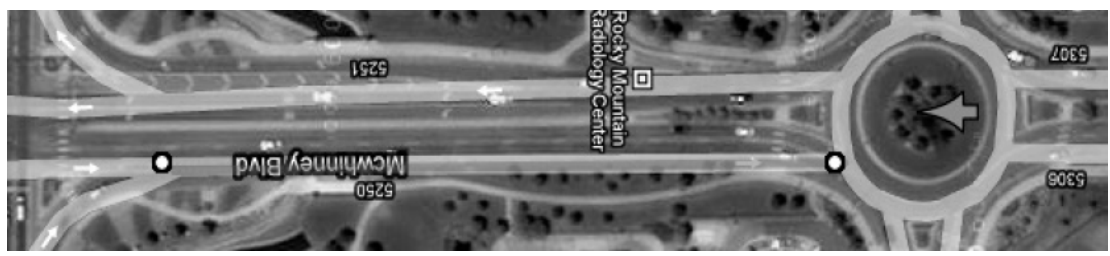

Figure 5: "Google Maps" view of roundabout approach. 


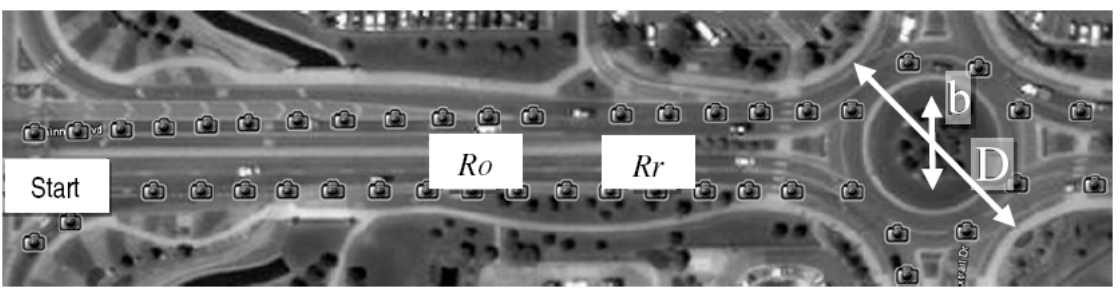

Figure 6: Locations of the "Streetview" images seen in Google Earth.

treatment, $b$, for both we view the roundabout in Google Earth in a planimetric (straight-down) view (Figure 6) and use its distance measurement tool.

In Google Earth we then jump "into" a Streetview location around $300 \mathrm{~m}$ away from the roundabout ("Start" in Figure 6). We orient the camera to simulate the view of a car driving towards the roundabout and jump to the next Streetview location ahead until the roundabout is reached.

During this journey, we record the locations: a) for the first indication of an obstacle; and b) for the certain recognition of a roundabout. Later, we determine the distances from these Streetview locations to the roundabout, $R o$ and $R r$, again from a plan view. Finally we jump into a Streetview location around 30 meters away, center the camera on the roundabout and using a ruler, measure the width of the base and the height of the treatment on the computer display.

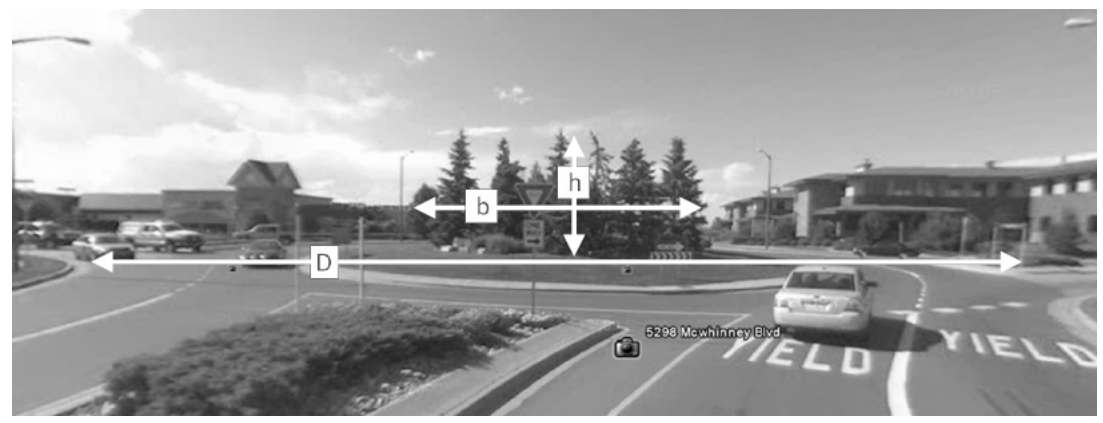

Figure 7: "Streetview" view of roundabout approach (large, compressed, 4 arm).

This allows us to calculate the treatment's true height, $h$, in meters (Figure 7), its apparent area $(A=b \times h)$, and its width to height ratio, $b / h$. Using the treatment's apparent area, $A$, we calculate the solid angles for the distances $R o$ and $R r$. We subjectively classify the roundabout's treatment type as reduced, compressed or slender. If a roundabout has more than one suitable approach, this procedure is repeated for each approach.

We performed linear regression to determine the relationship between the three geometry-based properties (the solid angle, $\Omega$; the base width-to-height ratio, $b / h$; the apparent area, $A$ ) and the two human-derived perceptibility distances $\left(R_{o}\right.$ and $\left.R_{r}\right)$. As may be expected, $R_{o}$ is generally better explained than 


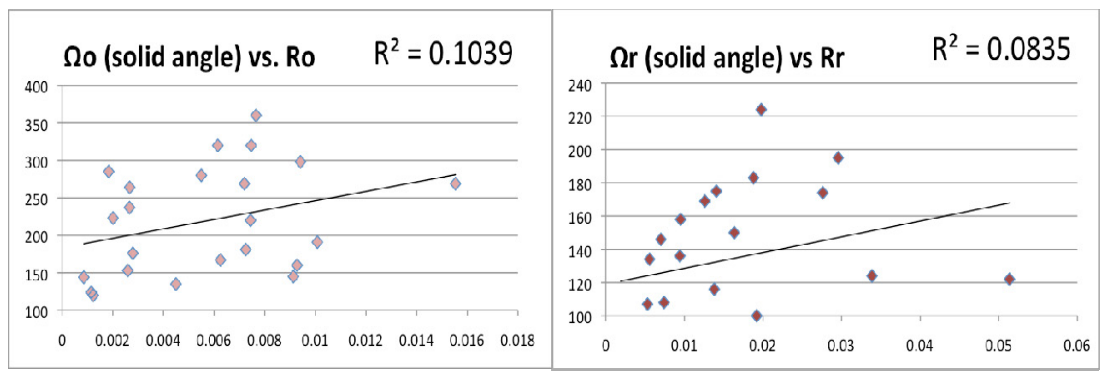

Figure 8: $\quad$ Linear regression for perceptibility (distance, in meters, y-axis) vs. solid angle (x-axis).

$R_{r}$ by our objective measures of perceptibility (area, $A$, essentially explains both equally well). The correlations between perception distances and solid angle $\Omega$ are relatively weak, explaining only around $10 \%$ of their relationship (Figure 8).

The correlations of the base width-to-height ration $(b / h$, Figure 9$)$, and of the apparent area $A$ (the profile of the treatment, Figure 10), are higher, which seems to indicate that they are better suited to capture the relationship between a treatment's geometric properties and a drivers ability to perceive it.
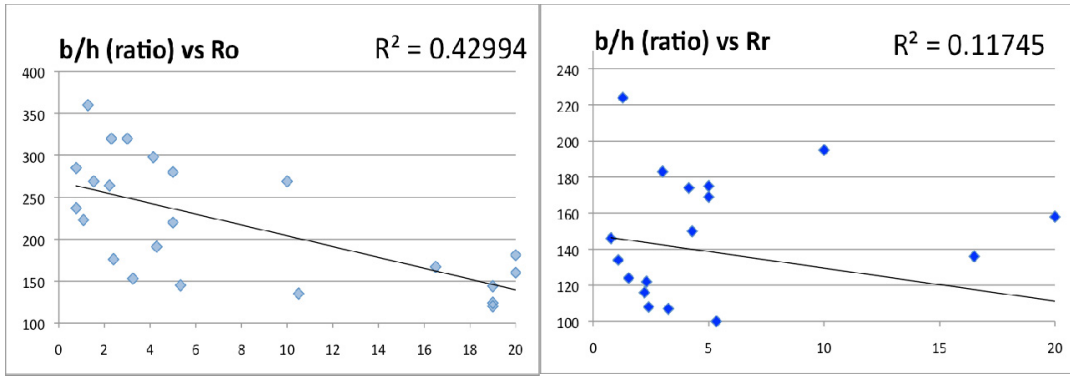

Figure 9: Linear regression for perceptibility (distance, in meters, y-axis) vs. width to height ratio (x-axis).
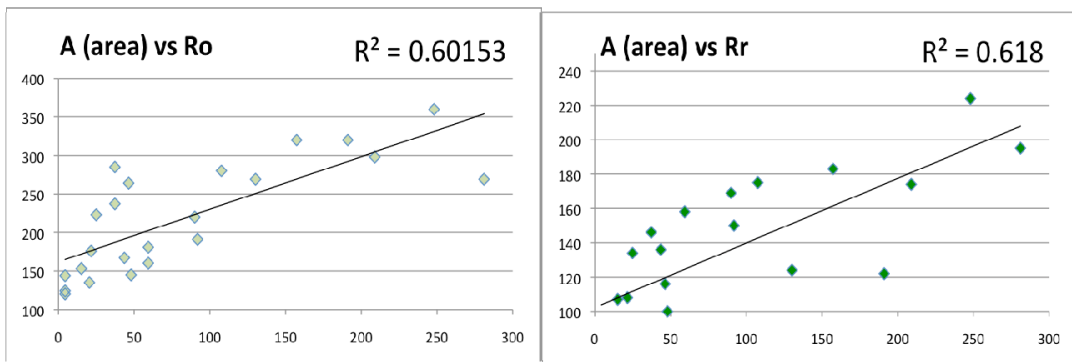

Figure 10: Linear regression for perceptibility (distance, in meters, y-axis) vs. apparent area (x-axis). 
While the base width-to-height ratio $b / h$ seems to be useful for indicating an upcoming obstacle (potentially a roundabout), the apparent area of a roundabout's treatment (i.e., base width $\times$ height) seems to be reasonably high factor in both, the first indication of an upcoming obstacle and the recognition of this obstacle as a roundabout.

The results of this proof-of-concept research indicates that some of the geometric attributes of a roundabout's treatment appear to be related to their perceptibility as measured through the "virtual" method demonstrated here. Further exploration of such relationships and the improvement of the method, would help in the planning of different roundabout scenarios by predicting its treatment's effectiveness.

Although use of Google Earth and Google Streetview demonstrates the potential of using a "virtual" visual experience for classification and measurement of road features, our current proof-of-concept approach imposes several types of limitations.

Determining the two perception distances $R_{o}$ and $R_{r}$ should be based on a more rigorous, formal user study, for which the current work may serve as a pilot study. Google Streetview's images are only of a moderate resolution and using a sequence of images taken every $15 \mathrm{~m}$ provides only a limited form of the true visuals a human driver would experience.

Ideally all approaches should show comparable visual conditions. Instead of showing the Google Streetview images on a single display, they could be projected one or more large screens (possibly dome-shaped), to provide a larger field of view. Alternatively, video logs or in-car video recordings could be used instead of Google Streetview. Ideally the user study should be based on a 3D computer graphics (virtual reality) simulation, which would permit the full control of all visual factors.

\section{Conclusions}

Factors related to visibility, perception and identification of road space significantly affects driver's behaviour and then road safety. It is most important for drivers entering a roundabout looking to the left and right, not through the central island. This fact allows beneficial use of the space in the central island whose development may enforce the warning perception of roundabout presence.

This last factor is important because to safely negotiate the roundabout the driver must clearly perceive in and understand the permitted maneuvers before reaching the intersection. In order to test the visual perception degree of roundabouts, we have developed two different experimental approches that try to match geometric concepts with a measure of visual perceptibly as a way of establishing how well a driver perceives different types and profiles of treatments.

At the present time, our research work is in progress. On one side, our goal is in testing and calibrating a roundabout classification into three types related to the central island development (reduced, compressed and slender). This way, the 
“on-site stand-by/2D" approach have been set up and data are under collection on a large sample of different roundabouts using a laser rangefinder.

On the other side, the pilot study results obtained with the "virtual video/3D" or proof-of-concept approach indicates that some of the geometric attributes of a roundabout's treatment appear to be related to their driver's perceptibility. Nevertheless, further exploration of such relationships and the improvement of the suggested methods are required.

\section{References}

[1] NCHRP, Roundabouts in the United States, Web Only Document 94: Appendixes to NCHRP Report no. 572, TRB, 2006.

[2] CETUR - Centre d'Etudes des Transport Urbains, Guide carrefours urbains, Bagneaux 1999.

[3] Pratelli, A. and Souleyrette, R. R., Visibility, Perception and Roundabout Safety, Urban Transport XIV, ed. Brebbia, WIT Press, pp. 577-588, 2009.

[4] Pratelli, A., Design of modern roundabouts in urban traffic systems. Urban Transport XII, eds. Brebbia and Dolezel, WIT Press, pp. 83-93, 2006.

[5] McShane, W.R., Roess, R.P., Traffic Engineering, Prentice Hall, 1990.

[6] FHWA, Manual on Uniform Control Devices, U.S. Department of Transportation, Washington, 2009 ed.

[7] SN 640263 - Swisse Norme, Carrefours giratoires, VSS, Zurich 2000.

[8] Hopkinson, R.G., The evaluation of visual intrusion in transport situations, Traffic Engineering \& Control, Dec. 1972, pp. 387-395.

[9] http://en.wikipedia.org/wiki/Solid angle

[10] Gordon, I. E., Theories of Visual Perception, Psychology Press, 2004. 